\title{
Intelligent reservoir operation system based on evolving artificial neural networks
}

\author{
Paulo Chaves, Fi-John Chang* \\ Bioenvironmental Systems Engineering, National Taiwan University, Taipei, Taiwan, ROC
}

\section{A R T I C L E I N F O}

\section{Article history:}

Received 17 December 2007

Received in revised form 12 March 2008

Accepted 16 March 2008

Available online 26 March 2008

\section{Keywords:}

Artificial intelligence

Reservoir operation

Evolving neural networks

Intelligent system

\begin{abstract}
A B S T R A C T
We propose a novel intelligent reservoir operation system based on an evolving artificial neural network (ANN). Evolving means the parameters of the ANN model are identified by the GA evolutionary optimization technique. Accordingly, the ANN model should represent the operational strategies of reservoir operation. The main advantages of the Evolving ANN Intelligent System (ENNIS) are as follows: (i) only a small number of parameters to be optimized even for long optimization horizons, (ii) easy to handle multiple decision variables, and (iii) the straightforward combination of the operation model with other prediction models. The developed intelligent system was applied to the operation of the Shihmen Reservoir in North Taiwan, to investigate its applicability and practicability. The proposed method is first built to a simple formulation for the operation of the Shihmen Reservoir, with single objective and single decision. Its results were compared to those obtained by dynamic programming. The constructed network proved to be a good operational strategy. The method was then built and applied to the reservoir with multiple (five) decision variables. The results demonstrated that the developed evolving neural networks improved the operation performance of the reservoir when compared to its current operational strategy. The system was capable of successfully simultaneously handling various decision variables and provided reasonable and suitable decisions.
\end{abstract}

(c) 2008 Elsevier Ltd. All rights reserved.

\section{Introduction}

Artificial intelligence $(\mathrm{AI})$ is a state-of-the-art technology that resembles the human thinking process in decision making and strategy learning. It has been well recognized for its outstanding ability to handle complex systems $[17,19]$ and has been adopted throughout the technology industry, providing the heavy lifting for logistics, data mining, medical diagnosis and many other areas [25]. In the last decade, AI techniques, such as artificial neural networks (ANN), genetic algorithms (GA) and fuzzy theory have also been increasingly applied to tackle some of the issues related to hydrological and water resources systems [7,9,20]. Recently, some attempts have been made to address storage reservoir optimization and operation through AI techniques. Raman and Chandramouli [24] and Chandramouli and Raman [4] proposed the use of ANN to generate operational strategies trained based on the optimal results from a deterministic Dynamic Programming (DP) model, for the case of a single and multiple reservoir system, respectively. Cancellier et al. [3] developed a neural network model to derive the operational strategies for an irrigation supply reservoir. In an attempt to take into account the stochastic characteristics of in-

\footnotetext{
* Corresponding author. Tel.: +886 2 23639461; fax: +886223635854.

E-mail addresses: phchaves@yahoo.com (P. Chaves), changfj@ntu.edu.tw (F.-J. Chang).
}

flow, Ponnambalam et al. [23] trained an adaptive neuro-fuzzy inference system (ANFIS) based on the optimal results obtained by another stochastic optimization model. Chang et al. [6] used a GA-based model to search for the optimal sequence of reservoir water releases, and then used these results as the training pattern of an ANFIS model. The AI techniques are also the main methods that have been used for the development of intelligent systems (IS) and decision support systems (DSS) in water resources management [16,27].

The references given above are similar in the sense that they first identify the "optimal target vector" (e.g., reservoir water releases) based on GA, DP or other optimization methods. Only then do they train an ANN-based model, using previously identified optimal values, to develop an intelligent operation system. These ANN-based models were used in an attempt to mimic the already "optimal" values for making new decisions in the future. However, the process of finding the initial optimal results and then training the ANN might face some drawbacks. In the case of optimization by DP, an increase in the number of decision and state variables could make the problem infeasible due to the curse of dimensionality [18]. In the case of optimization by GA, each decision variable at each stage has to be assumed as a parameter to be optimized. Hence, for long optimization horizons, the number of parameters to be optimized is proportional to the number of stages. Finally, for this two-step process, the error metric used in 
the training of the ANN-based model is not directly associated with the operational objectives, which could therefore affect the operation efficiency and performance.

In this paper, we have developed an intelligent system based on an evolving ANN model and then applied it for the optimizing operation of a multi-purpose reservoir. Evolving in this case means that the parameters (weights and bias) of the ANN model are identified (optimized) by the GA evolutionary optimization technique. Accordingly, the ANN model should represent the operational strategies of reservoir operation. As the GA model is responsible for the optimization of the ANN parameters instead of decision values, only a fixed number of parameters need to be identified independently of the size of the optimization horizon. This can be seen as a kind of parameterization of the operational strategies. Moreover, to handle multiple decision variables, the proposed model tends to be more feasible than traditional methods, such as GA and DP, as these variables can be accounted as new ANN output units. The addition of new output units does not necessarily compromise the feasibility and optimality of the new method, as GA is able to optimize high order and complex networks [28]. Finally, the proposed intelligent system presents great potential to be combined with other prediction models as the GA-based training can be implemented in a forward-moving direction and handle complex systems in a flexible way.

To test the performance of the developed intelligent reservoir operation system, we applied it to the actual operation of the multi-purpose Shihmen Reservoir, located in North Taiwan, considering 10-day time steps. The Reservoir was operated for multiple uses, such as domestic water supply, agricultural uses, environmental minimum flow requirements and hydropower generation. The multiple objectives of the reservoir were evaluated by using fuzzy membership functions as an effective way to normalize and to aggregate different types of objectives. To validate the optimality of the proposed ENNIS, first we compared its results with those obtained by a DDP model, considering single objective (energy production). Then, the problem was set in a more complex manner, in which five decision variables were simultaneously handled. We also tested the influence of various types of input information of the ANN operation model.

\section{Methodology}

Traditionally, in training an ANN-based model, input-output patterns are normally necessary. For the case of system control by ANN, these target vectors may be obtained from previously operational results. For example, in the case of reservoir operation, past operating water releases may be used as the target training vector. However, training the ANN model with such values does not guarantee that the ANN will yield an optimal operational strategy. That is why previous researches have applied other optimization techniques for finding such optimal input-output patterns before training the ANN models [6]. In this study, we present a novel approach, in which the ANN model are directly trained by GA using an objective function, instead of the error metrics between calculated output and target values. Moreover, the proposed methodology can be easily extended to the optimization and control of other water resources systems. In the following sub-sections we present the main features of two of the techniques used here (GA and ANN) and the proposed Evolving ANN Intelligent System (ENNIS) for reservoir operation.

\subsection{Genetic algorithm}

Evolutionary computation applies biologically inspired concepts such as populations, mutation and survival of the fittest to generate increasingly better solutions to the problem. Even though these models may not guarantee the "optimal" solution, they are usually capable of achieving near global optimal solutions to problems where traditional models would fail to converge due to dimensionality and local optimal related problems. One of the most popular evolutionary optimization techniques is surely the genetic algorithm (GA). In recent years, GA has been widely used to solve a great number of hydrological and water resources related problems $[5,26]$. Some of the advantages of GA models are (a) a powerful capability to deal with a non-linear system global optimization, and (b) a robust ability to solve highly non-linear, non-convex problems. GA may also become a very efficient technique for tackling the dimensionality problem when combined with other optimization techniques, such as in Huang et al. [12], where GA was combined with DP for the optimization of a multireservoir system.

However, GA presents some disadvantages: (a) the difficulty of explicitly accounting for constraints and maintaining feasible solutions in the population, (b) an increase in the number of variables proportional to the size of the optimization horizon, as the variables of each time step become a state or decision variable, aggravating the convergence problem. Some of these problems may be overcome if operational strategies are parameterized in some way, such as the one developed in this study.

Normally, GA uses four main types of operators to create, at each new GA generation, a new GA population based on the previous one. These operators are elitism, selection, crossover and mutation. Elitism operators can guarantee that the best individuals of a population will proceed automatically to the next generation. Selection operators are responsible for the selection of individuals (the so-called "parents") in which the following two operators are applied. Crossover operators combine two "parents" to form children for the next generation. And finally, mutation operators which apply random changes to individual "parents" to generate "children" for the next generation. By trial and error, we identified the "best" setting and parameters for our GA model as follows: number of elitism individuals was set equal to five, total population size around 200 individuals, "Rank" scaling fitness function and "Blend" and "Scattered" crossover functions. Other parameters used for the GA model are "Simple" and "Non-uniform" mutation functions, variable crossover fraction ranging from 0.75 to 0.95 depending on the number of the generation and "triangular probability" selection function. The two methods for each kind of operator were applied in a random way for each generation, which has proven to greatly increase the efficiency of the GA model, when different testing functions are used.

\subsection{Artificial neural networks operation model}

Artificial neural networks (ANNs) are known as flexible modeling tools with capabilities of learning the mathematical mapping between input and output variables of non-linear systems and to generalize for control, classification and prediction. They are capable of providing a neuro-computing approach for solving complex problems that might otherwise not have a tractable solution. In the last decade, ANNs have been widely applied with success to various water resources problems, such as rainfall-runoff modeling [8], rainfall forecasting [21], ground water problems [14] and reservoir operation problems $[10,13]$. A review of the application of ANNs to hydrology can also be found in the ASCE Task Committee report $[1,2]$. The following are advantages of artificial neural networks (ANN)-based models for reservoir operation: (i) no mathematical formulation is required to build the model; therefore, the modeling approach is in most cases fast and flexible; (ii) ANN is a data driven approach, which can efficiently handle non-linear relationships, and (iii) the experts' and users' experience may be 
easily incorporated into the model development through the addition of input and output neurons.

Some of the important features when developing a ANN-based model for reservoir operation are (i) the type of ANN (e.g., feedforward, neuro-fuzzy); (ii) the necessary input information (e.g., inflow, storage, demand, rain); (iii) the output information (e.g., multiple output networks, storage variation, water releases) and (iv) inflow situation (e.g., previous, average, expected, forecasted values). How these issues influence the performance of the model may vary from case to case depending on the characteristics of the system under consideration, the operation objectives and the available data and information.

To apply our intelligent system to the Shihmen Reservoir, we investigated all the above mentioned features. The ANN model was based on feedforward networks with three layers (input, hidden and output layers) connected through hyperbolic tangent and linear transfer functions, respectively. The final architecture of the built ANN operation model is shown later in Figs. 6 and 7, for the two cases of single and multiple decision variables, respectively.

\subsection{Evolving ANN Intelligent System (ENNIS) for reservoir operation}

Based on an evolving ANN, we develop an intelligent system for reservoir operation, which has two phases, the training (which also could be seen as an optimization phase) and the testing (operation phase) periods. Fig. 1 shows the overall flowchart. The operational objectives are first defined. Then, a set of parameters for the ANN, such as the number of hidden units and type of input and output, are assumed and the basic (not-yet-trained) architecture of the ANN is built. Next, the training process of the ANN weights through the genetic algorithm starts. For the first GA generation, an initial (random) population is constructed having each individual of the GA population representing a set of ANN parameters (e.g., weights and bias). For the following GA generations, GA operators are responsible for evolving the subsequent populations.

Having decided a set of parameters for the ANN operation model (here constructed as shown in Figs. 6 and 7), it is possible to obtain the values of the decision variables, as its input information is known at the beginning of each stage $t$. For continuity purposes the reservoir mass balance function is defined as shown in

$R_{t}=S_{t-1}-S_{t}+I_{t}$

where $S$ are the storage levels, $R$ indicates release and $I$ represents inflow for each time $t$. Although we neglected losses such as evaporation, these losses can be easily incorporated into the mass balance function without compromising the proposed methodology.

If the ANN operation model recommends a certain decision that may result in an infeasible state condition, such as negative storage, some constraints may be applied, forcing the reservoir system to stay at a certain desired level. Then, based on these minimum and maximum values, the decisions are recalculated by using the inverse of the system state function, for example, the reservoir mass balance equation. On the other hand, if the resulting condition is physically feasible but still undesired, some penalty coefficient may be introduced.

To train ANN models in constructing an intelligent system, "optimal target vectors" have been optimized beforehand by observed values or other optimization techniques [7]. However, in our case, the training is proposed to be based on the direct maximization (minimization) of the objective function proposed by reservoir operators. The objective function can be formulated as in Eq. (2), for a certain training operational horizon $\mathrm{Tr}$. The optimized parameters (ANN weights and bias) are expected to give the maximum value for the objective function when the reservoir is operated by using the ANN operation model. The final value of the objective function of Eq. (2), considering the whole optimization

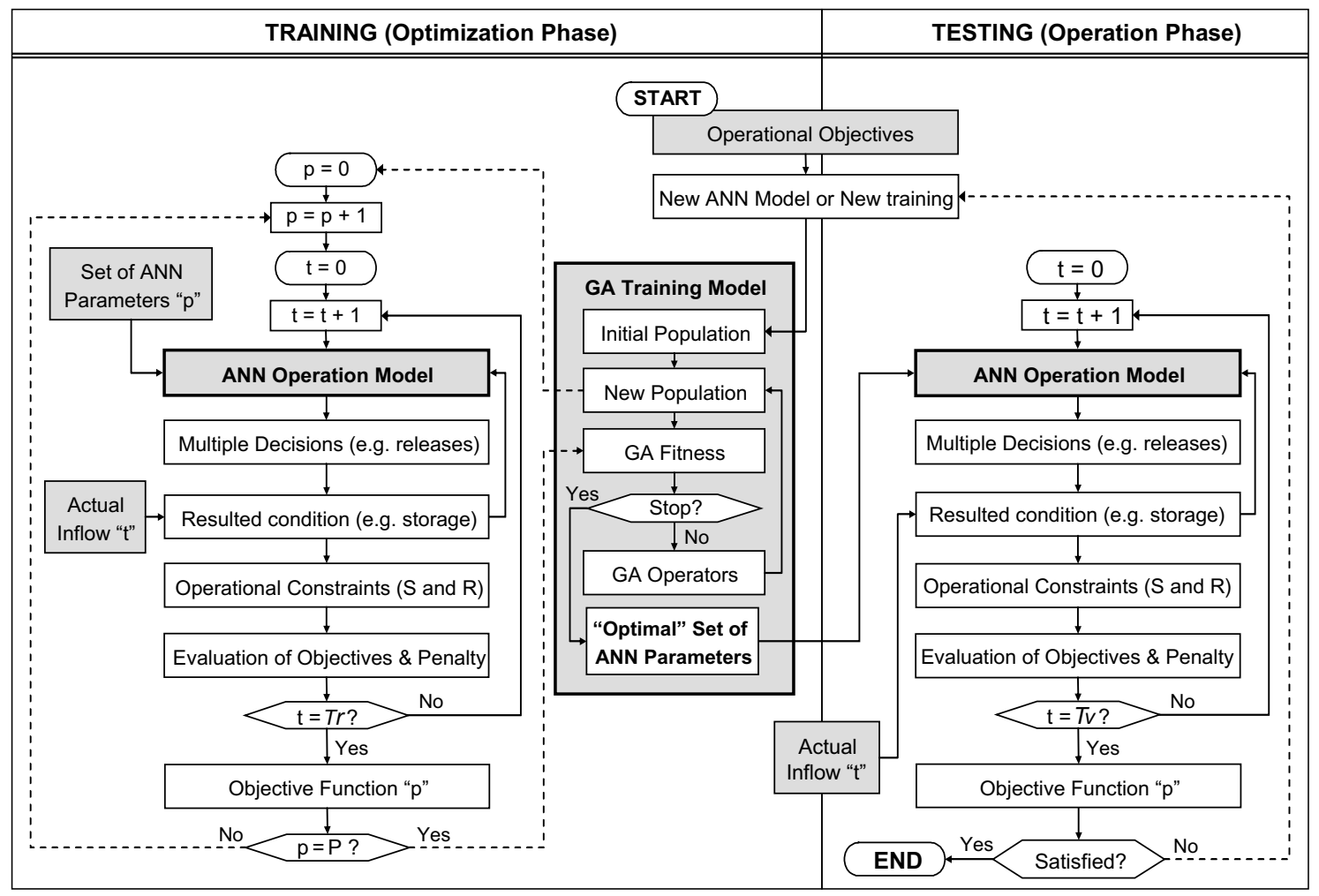

Fig. 1. Flowchart for the development of evolving ANN-based intelligent systems for reservoir operation. 
(training) horizon, is associated with the fitness of each set of parameters (ANN weights) in the GA model:

$F=\max \left[\sum_{t=1}^{T r}\left(E_{t} \alpha_{t}\right) \cdot \delta\right]$

where $E_{t}$ is the aggregation of all evaluated objective values for each stage (time step) $t, \alpha_{t}$ is the weight for each time step; here we considered all the weights to be the same and equal to $1 / T r$. Nevertheless, different weights may be incorporated to increase the importance of a particular period in time or as a discount factor, where $\operatorname{Tr}$ is the total number of time intervals within the training operation horizon. Here, the summation of $\alpha_{t}$ is equal to 1 , which guarantees the final value of the objective function (2) to be between $[0,1]$. Finally, $\delta$ is a penalty coefficient that may be incorporated to improve the GA training process. Here the proposed penalty coefficient is defined as in

$\delta=\frac{T-P}{T}$

where $P$ is the penalties, in other words, the number of times the system is considered to have failed during the operational horizon $T$, based on certain constraints, such as minimum and maximum values of water releases and storage volumes. Here the failure of the reservoir operation was considered to occur whenever final storage volume was less than $0.045 \mathrm{~km}^{3}$ or total water release from reservoir was less than $5 \mathrm{~m}^{3} / \mathrm{s}$.

The aggregated evaluation value, $E_{\mathrm{t}}$, may be defined according to the kind of aggregation operator to be used. There are a variety of operators in the literature used for the aggregation of multiple objectives [15]. The choice of which operator to use will most depend on the user and the problem being formulated. The most common aggregation operators are the weighted-average, the product and the minimization. Eq. (4) shows the mathematical formulation of the weighted-average operator, which is the one used later for our case study applications:

$E_{t}=\sum_{n=1}^{N} E_{n} \cdot \beta_{n}, \quad$ where $\sum_{n=1}^{N} \beta_{n}=1$

where $\beta_{n}$ is the weight corresponding to each objective $n$, which may vary depending on the importance of each operational objective. $N$ is the total number of objectives. $E$ can be represented by fuzzy membership evaluation functions (such as the ones shown later in Fig. 4), which vary from 0 to 1 , from completely not satisfied to fully satisfied.

As for the stopping criterion of the GA training model, the two most popular methods are the maximum expected value of the performance function and the total number of generations. Besides these two criteria, we have implemented a stopping rule for no significant changes on the objective function after a certain number of generations, and the training dataset is split into two sets for which a cross-validation was considered. For this cross-validation, the GA training will be stopped whenever a persistent decrease in the value of the objective function (OF) of the second dataset is observed, regardless of the increase of the objective function of the first set. The latter criterion intends to avoid problems of overtraining the ANN operation model. Accordingly, until a stopping criterion is reached, the GA model is responsible for evolving the parameters (in our case the weights and bias of the ANN operation model) based on GA operators. Moreover, the fitness value used in the GA model is here based on the objective function itself of the reservoir operation, which should be defined by reservoir operator authorities.

Once the "optimal" weights have been identified (optimized) by the GA model, the ANN operation model is used to operate the reservoir over the testing horizon $T v$. The operation performance is evaluated based directly on the achievement of the operational objectives measured by the value of the OF. If the set of assumptions taken for the ANN model (e.g., hidden units and input variables) is considered satisfactory, the model is ready for actual application; if not, the process starts again with new set of assumptions for the ANN model and also different evaluation functions.

\section{Application}

\subsection{Study area}

The Shihmen Reservoir is located in the upstream portion of the Tahan River basin, North Taiwan (Fig. 2). The Reservoir is operated for multiple purposes, including water supply for irrigation, industrial and domestic uses, flood control, generation of hydropower energy and recreation. The watershed area is $763.4 \mathrm{~km}^{2}$. Normal variation of storage volumes may occur between $0.0215 \mathrm{~km}^{3}$ (dead volume) and $0.2620 \mathrm{~km}^{3}$ (maximum expected storage). Maximum expected and warning water levels are at 245 and $249.5 \mathrm{~m}$, the latter corresponding to a storage volume of approximately $0.300 \mathrm{~km}^{3}$.

\subsection{Shihmen Reservoir operation objectives}

To be able to train the ANN operation model even without an "optimal target vector", as we propose here, it is necessary to define operational objectives. The operational objectives defined here are related to the water uses and water rights considered in the actual operation of the Reservoir at the present. The objectives have been formulated as fuzzy membership evaluation functions, normalized between 0 and 1 , representing the degree of satisfaction at which each objective is fulfilled. In our case study, the values calculated for each degree of satisfaction are then aggregated by using Eq. (4), the weighted-average operator. Fig. 3 shows

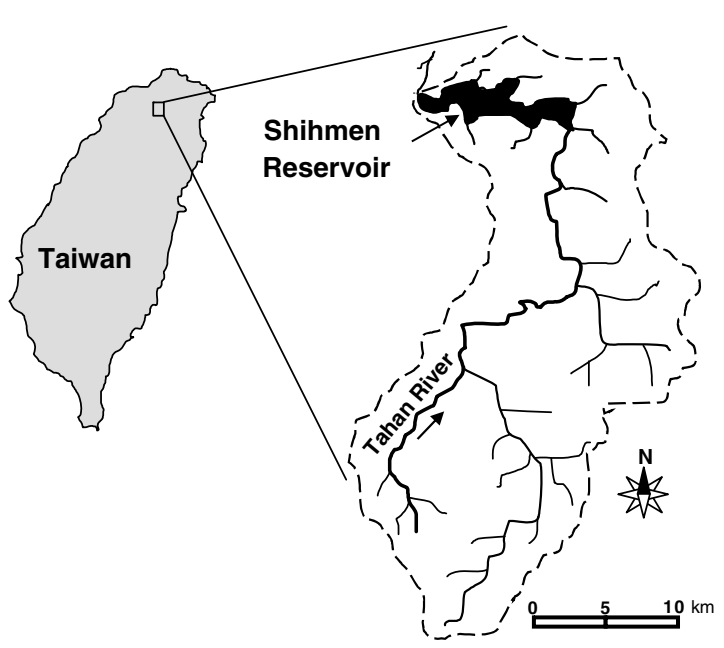

Fig. 2. Location of Shihmen Reservoir watershed.

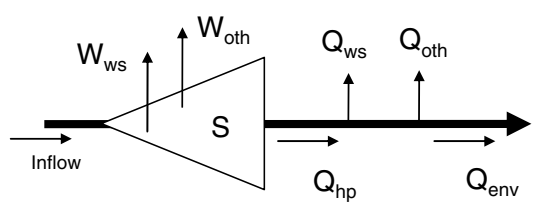

Fig. 3. Schematic representation of the Shihmen Reservoir with different water uses. 
Table 1

Monthly demands considered for the operation of Shihmen Reservoir

\begin{tabular}{|c|c|c|c|c|c|c|c|c|c|c|c|c|c|c|}
\hline \multicolumn{3}{|l|}{ Type of demand } & \multicolumn{12}{|c|}{ Demand values of Shihmen Reservoir for each month } \\
\hline Description & Symbol & Unit & January & February & March & April & May & June & July & August & September & October & November & December \\
\hline Domestic use upstream & $D W_{\text {ws }}$ & $\mathrm{m}^{3} / \mathrm{s}$ & 4.3 & 3.5 & 3.5 & 3.5 & 3.5 & 3.5 & 3.5 & 3.5 & 3.5 & 3.5 & 3.5 & 4.3 \\
\hline Other uses upstream & $D W_{\text {oth }}$ & $\mathrm{m}^{3} / \mathrm{s}$ & 0.07 & 3.5 & 5.4 & 4.8 & 3.9 & 4.5 & 6.0 & 5.7 & 5.1 & 6.0 & 4.4 & 0.07 \\
\hline Hydropower generation & $D Q_{\mathrm{hp}}$ & $\mathrm{m}^{3} / \mathrm{s}$ & 0.0 & 1.1 & 9.1 & 6.8 & 7.3 & 20.6 & 20.7 & 30.2 & 21.7 & 22.7 & 5.2 & 1.6 \\
\hline Domestic use downstream & $D Q_{w s}$ & $\mathrm{~m}^{3} / \mathrm{s}$ & 4.2 & 4.8 & 4.8 & 4.8 & 3.7 & 3.7 & 3.7 & 3.7 & 3.7 & 3.7 & 4.1 & 3.6 \\
\hline Other uses downstream & $D Q_{\text {oth }}$ & $\mathrm{m}^{3} / \mathrm{s}$ & 0.15 & 5.4 & 7.9 & 6.6 & 5.3 & 6.4 & 8.2 & 8.2 & 6.8 & 7.9 & 6.1 & 0.15 \\
\hline Environ. minimum flow & $D Q_{\text {env }}$ & $\mathrm{m}^{3} / \mathrm{s}$ & 7.0 & 7.0 & 7.0 & 7.0 & 7.0 & 13.0 & 13.0 & 13.0 & 13.0 & 13.0 & 7.0 & 7.0 \\
\hline
\end{tabular}

Note: "Other uses" include mainly demands for agricultural activities.

a schematic representation of the reservoir system with its water releases and withdraws for the respective water uses (operational objectives). Accordingly, Table 1 shows the monthly demand values being used for the present operation of Shihmen Reservoir.

Note that the environmental minimum flow is not an official water use required in the present reservoir operational strategy. However, it has been identified by some environmentalists as crucial for the sustainability of aquatic life and good environmental conditions downstream of the Reservoir [22]. Therefore, environmental minimum flow was also considered as one of our objectives. Furthermore, as a 10-day time step (each month is divided into three steps, i.e., the first 10 days, the second 10 days, and the rest of days in a month, respectively) was adopted for our operation, flood-related constraints and risks were not considered as objectives. However, this should be taken into consideration when smaller operational time scales are used (e.g., hours to days). To define the importance (priority) of each objective, we considered the present operation of the Reservoir (as shown in the next sub-section). Hence, as the objectives present different importance, various shapes for the fuzzy membership evaluation functions were proposed, as shown in Fig. 4 :

$E_{n}=\frac{Q_{n}}{D Q_{n}}$

$E_{n}=\left(\frac{Q_{n}}{D Q_{n}}\right)^{a_{n}}$

$E_{n}=1-\left(1-\frac{Q_{n}}{D Q_{n}}\right)^{b_{n}}$

where $E$ is the evaluation function for each water use $n$. $Q$ (or $W$ for the variables of the upstream part of the reservoir) is the actual volume of water supplied and $D Q$ ( $D W$ for upstream) is the required demand; $a_{n}$ and $b_{n}$ are coefficients used to increase and decrease

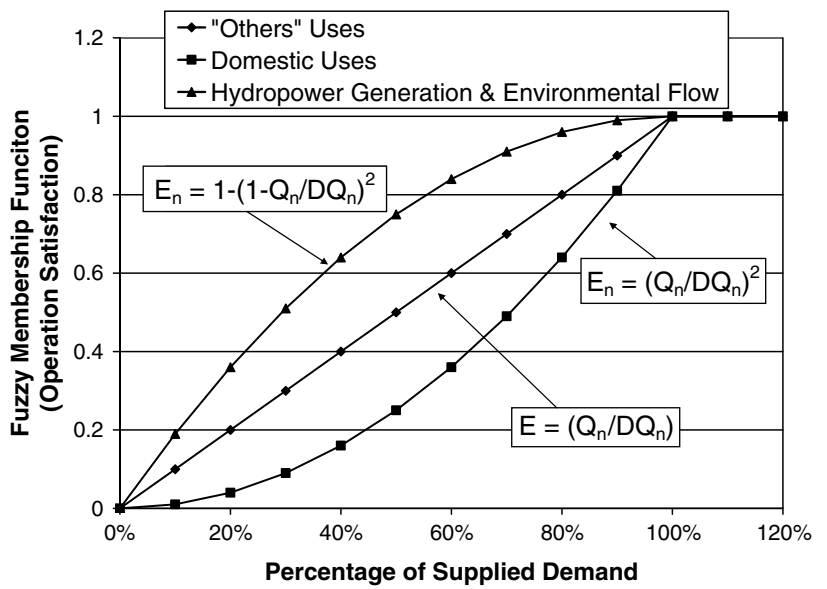

Fig. 4. Fuzzy membership functions considered for evaluation of reservoir objectives. the priority of each use $n$, respectively. These coefficients may be adjusted by trial and error considering the results obtained after training and testing of the ANN operation model. For our case study, both $a$ and $b$ were assigned as being equal to 2 , resulting in the fuzzy membership functions shown in Fig. 4. Nevertheless, the proposed methodology may also handle other shapes of membership functions. Eq. (5) was considered for upstream and downstream water supply related objectives, whereas Eq. (6) was applied for the fuzzification of the other four.

The evaluation function for each water use was based on the percentage of the actual supplied water over the total required demand. The demands $D Q$ (and $D W$ ) vary for each time step according to the values listed in Table 1 . The actual supplied volumes were calculated based on the output units of the ANN operation model and based on the actual available water for each specific stage, as explained in the next sub-section.

\subsection{Shihmen Reservoir existing operational strategies (the M-5 rule curves)}

In Taiwan, most reservoirs are operated according to rule curves. According to the rule curves, the Reservoir is mainly operated based on the time of decision, the present water level and water demands. These demands are related to domestic/industrial uses (index "ws"), agriculture/other uses (index "oth") and hydropower generation ("hp"), as shown in Table 1 . Note as well that $Q$ and $W$ represent the water uses for downstream and upstream parts of the reservoir, respectively (Fig. 3). The Shihmen Reservoir rule curves (also known as M-5 curves) are a simple way to operate the reservoir. For Shihmen Reservoir there are three basic rule curves, UC, LC and CC (upper, lower and critical curves, respectively, as shown in Fig. 5). To make the results after operation by our intelligent system comparable, we also operated the reservoir following the existing operational M-5 rule curves. The only modification implemented here, which differs from the original

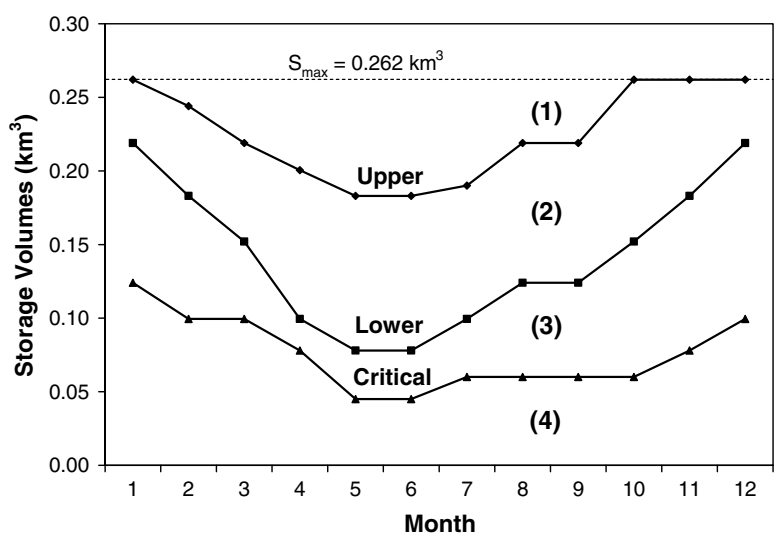

Fig. 5. Existing operational rules of Shihmen Reservoir: the M-5 rule curves. 
Table 2

Operational strategy of Shihmen Reservoir using the M-5 rule curves

\begin{tabular}{|c|c|c|}
\hline No. & Condition & Operational decision \\
\hline (1) & $\begin{array}{l}\text { If storage > upper curve } \\
\text { (UC) }\end{array}$ & $\begin{array}{l}\text { Hydropower water release }\left(Q_{\mathrm{hp}}\right) \text { increases to } \\
\text { keep storage levels }=\mathrm{UC} \text {. All the other } \\
\text { demands are fully supplied to the monthly } \\
\text { values shown in Table } 1\end{array}$ \\
\hline (2) & $\begin{array}{l}\text { If lower curve } \\
\text { (LC) < storage } \leqslant \text { upper } \\
\text { curve (UC) }\end{array}$ & $\begin{array}{l}\text { All demands are fully supplied to the monthly } \\
\text { values shown in Table } 1\end{array}$ \\
\hline (3) & $\begin{array}{l}\text { If critical curve } \\
(\mathrm{CC})<\text { storage } \leqslant \text { lower } \\
\text { curve }(\mathrm{LC})\end{array}$ & $\begin{array}{l}Q_{\mathrm{ws}}=D Q_{\mathrm{ws}} \text { and } W_{\mathrm{ws}}=D W_{\mathrm{ws}} ; Q_{\mathrm{oth}}=D Q_{\mathrm{oth}} \text { and } \\
W_{\mathrm{oth}}=D W_{\mathrm{oth}} ; Q_{\mathrm{hp}}=0.8 \times D Q_{\mathrm{hp}}\end{array}$ \\
\hline (4) & $\begin{array}{l}\text { If storage } \leqslant \text { critical curve } \\
(\mathrm{CC})\end{array}$ & $\begin{array}{l}Q_{\mathrm{ws}}=D Q_{\mathrm{ws}} \text { and } W_{\mathrm{ws}}=D W_{\mathrm{ws}} ; Q_{\text {oth }}=0.7 \times D Q_{\text {oth }} \\
\text { and } W_{\text {oth }}=0.7 \times D W_{\text {oth }} ; Q_{\mathrm{env}}=0.5 \times D Q_{\mathrm{env}} ; \\
Q_{\mathrm{hp}}=Q_{\text {oth }}+Q_{\mathrm{env}}+Q_{\mathrm{ws}}\end{array}$ \\
\hline
\end{tabular}

operational strategy, is the consideration of demands for environmental minimum flow $\left(Q_{\text {env }}\right)$. Based on the M-5 curves, there are four distinct operating policies (number in brackets in Fig. 5), which are schematically described in Table 2.

Based on the operational strategy using the M-5 curves, we may observe that "Domestic" uses (represented by the index ws) have preference over "Other" uses (oth) which have preference over hydropower (hp) and environmental minimum flow (env) demands. These points were also considered when developing our intelligent systems for the Shihmen Reservoir, supporting the decision on prioritizing the operational objectives.

Nevertheless, due to the high variability of natural streamflow and the wide gap between the M-5 curves, determining the "best" water release precisely according to the above rules alone is unlikely. A knowledgeable expert with a lifetime of experience in the operation of this reservoir would undoubtedly be helpful and cru- cial for the successful operation of the reservoir, especially under extreme events (e.g., long drought periods). However, expert experience and knowledge might not be easy to duplicate or either promptly available in all times. This also indicates the importance for the development of intelligent systems for reservoir operation, which usually tends to be less subjective.

\subsection{Developing ENNIS for operation of Shihmen Reservoir - Case 1: Single decision}

We first investigated the optimality of the proposed system in a simple manner for better illustration and compared its results to the ones obtained by a deterministic dynamic programming model. The problem was formulated to have a single objective, which is the maximization of energy generation. The final architecture of the developed ENNIS for Case 1 is shown in Fig. 6, where input variables are storage $\left(S_{t-1}\right)$, average inflow $\left(I_{\text {mean }}\right)$, total necessary water release for maximum energy production and, observed inflow at time $t$. Note that a case in which no observed inflow is given was also carried out, resulting in only three input variables. A decision on water release is given by the output neuron and through equations shown in Case 2.

The DP model was developed by also considering only one decision variable (water release) and one state variable (storage volume). The constraints of minimum and maximum total release and storage volumes, used for both models, were $10 \mathrm{~m}^{3} / \mathrm{s}<$ release $<2000 \mathrm{~m}^{3} / \mathrm{s}$ and $0.045 \mathrm{~km}^{3}<$ storage $<0.262 \mathrm{~km}^{3}$. The ENNIS model developed for the present case, illustrated in Fig. 6, shows the output decision as $y_{1}$, which corresponds to a percentage of the maximum discharge capacity of the hydropower $\left(140 \mathrm{~m}^{3} / \mathrm{s}\right)$. For Case 1, no penalty coefficient was considered for either model. Moreover, to investigate the influence of discretization on the DP model, the domain of the decision variable was discretized consid-

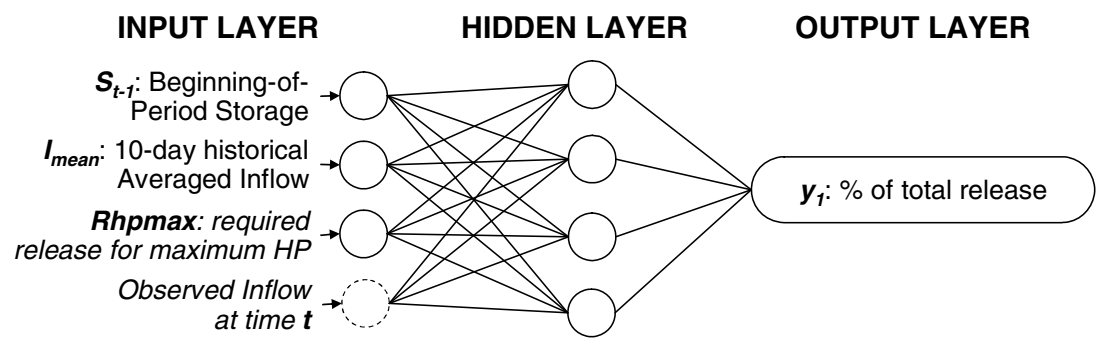

Fig. 6. Basic architecture of the ANN operation model - Case 1.

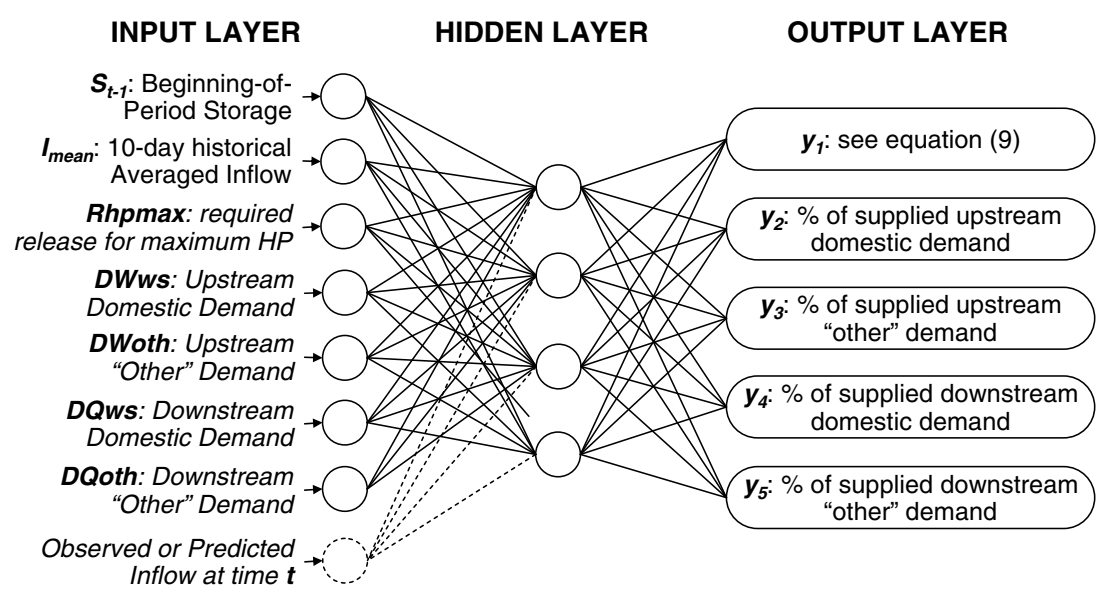

Fig. 7. Basic architecture of the ANN operation model - Case 2. 
ering 5 and 50 discrete intervals. The goal here was the optimization of hydropower generation of Shihmen Reservoir, making it a single objective problem. The general DP recursive formulation [11] is presented in the equation below:

$F_{t}\left(S_{t}\right)=\max _{S_{t+1}}\left\{E_{t}(\cdot)+F_{t+1}\left(S_{t+1}\right)\right\}$

where $F$ is the objective function and $E$ is the evaluation function, here defined as in Eq. (7). Note that the objective function used for both, ENNIS and DP, had the same optimization horizon under the same hydrological conditions and constraints.

\subsection{Developing ENNIS for operation of Shihmen Reservoir - Case 2: Multiple decisions}

For multiple decisions, the proposed ENNIS is also built as a single network with multiple outputs (five). Moreover, as in Case 1, the ANN model was based on feedforward networks with three layers. After a trial-and-error process and based on the available information about the system, we identified the basic structure of the ANN operation model, as shown in Fig. 7 for Case 2. The input layer is constituted by six or seven variables, each represented by one input neuron. The first two variables are initial storage and historical 10-day averaged inflow for each of the 36 intervals $i$ of oneyear period. The third variable to be considered is the water release necessary for maximum energy production, which is a function of the present water level and the maximum producible energy $(2000 \mathrm{MW}$ h). The other four input variables are the total demand for each water use for each month, as shown in Table 1. Moreover, for this case, we investigated not only the influence on the efficiency of the ENNIS operational strategy, if observed inflow information were available, but also the influence by the use of predicted inflow. For the sake of conciseness, no further discussion is given about the prediction model (based on a traditional feedforward multilayer perceptron ANN), but only its results are shown in Fig. 8 (the correlation coefficient between observed vs. predicted inflow is about 0.78 ). This also serves for investigating the behavior of the ENNIS model when handling noise and imprecise information.

The output layer presents five neuron units (decision variables), of which the first one $\left(y_{1}\right)$ corresponds to part of the total water volume to be supplied (released water for both upstream and downstream of reservoir uses), as shown in Eq. (9). The second $\left(y_{2}\right)$ and the third $\left(y_{3}\right)$ ones give the percentage of water to be supplied to domestic (ws) and other (oth) uses, respectively, for the upstream demands and uses. The outputs $y_{4}$ and $y_{5}$ are similar to the two previous ones but for the downstream demands. Then, knowing the total supplied water minus the volumes considered

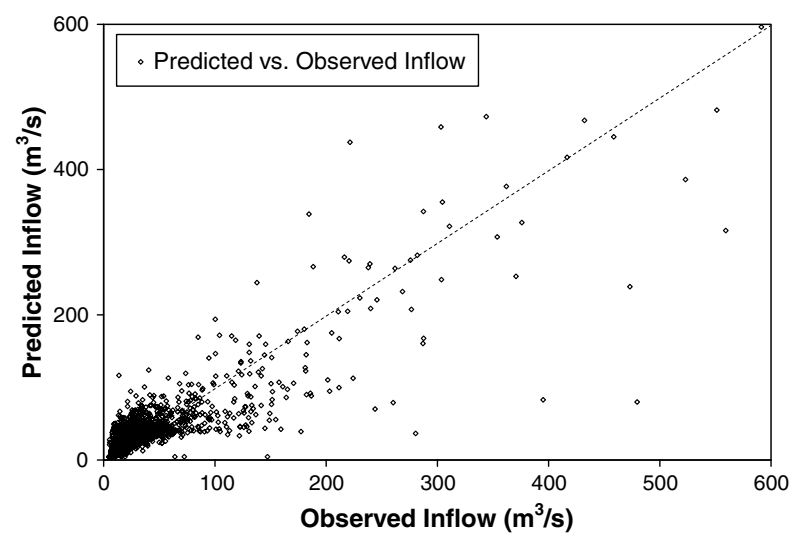

Fig. 8. Comparison of predicted vs. observed inflow into Shihmen Reservoir. for domestic and other uses, we calculate the available water for hydropower generation and the water left in the river accounting for the environmental minimum flow. The calculation of each water release was carried out using the following equations:

$$
\begin{aligned}
& Q_{\mathrm{total}, t}=10+140 \cdot y_{1, t} \\
& W_{\mathrm{ws}, t}=D W_{\mathrm{ws}, m} \cdot y_{3, t} \\
& W_{\mathrm{oth}, t}=D W_{\mathrm{oth}, m} \cdot y_{5, t} \\
& Q_{\mathrm{ws}, t}=D Q_{\mathrm{ws}, m} \cdot y_{2, t} \\
& Q_{\mathrm{oth}, t}=D Q_{\mathrm{oth}, m} \cdot y_{4, t} \\
& Q_{\mathrm{hp}, t}=Q_{\mathrm{total}, t}-W_{\mathrm{ws}, t}-W_{\mathrm{oth}, t} \\
& Q_{\mathrm{env}, t}=Q_{\mathrm{hp}, t}+Q_{\mathrm{spill}, t}-Q_{\mathrm{ws}, t}-Q_{\mathrm{oth}, t}
\end{aligned}
$$

where $y_{1}-y_{5}[0,1]$ are the ANN operation model output (decisions) and $m$ is an index representing the month of the operation stage. Other variables have been previously defined. In cases where there was not enough water to have the recommended $Q_{\text {total }}$, all demands were proportionally decreased by the ratio between the recommended total release and the available water in the reservoir. Moreover, for $Q_{h p}$ values calculated larger than the maximum intake capacity of the hydropower plant (here defined as $140 \mathrm{~m}^{3} / \mathrm{s}$ ), and reservoir capacity over the maximum determined value of $0.262 \mathrm{~km}^{3}$, all water surplus shall be released through the spillways $\left(Q_{\text {spill }}\right)$. Also, if future inflow were to overflow the reservoir due to insufficient recommended total release $\left(Q_{t o t a l}\right)$, the surplus of water was also considered to be released through the reservoir spillways. These assumptions were valid as the inflow used here is equal to the 10-day average. For shorter time-step periods when flood control is of greater importance, another output neuron could be introduced to represent, for example, the necessary spillway release. The release of water surplus was also considered for the operation of Shihmen Reservoir, when using the M-5 rule curves strategy. For the case study, failure of the reservoir operation was considered to occur whenever final storage volume was less than $0.0450 \mathrm{~km}^{3}$ or water left in the river (environmental flow) was lower than $2 \mathrm{~m}^{3} / \mathrm{s}$. Minimum and maximum allowed variation of the storage volumes was limited to 0.0215 and $0.2620 \mathrm{~km}^{3}$, respectively.

\section{Results}

The dataset was divided into two sets, a training horizon of 24 years (January 1964-December 1987) with 864 time intervals and a testing horizon of 17 years (January 1988-December 2004) with 612 time intervals also of 10 days. In the training phase, to avoid any problems related to the variability of the GA operator, due to its random characteristics, for each ANN model structure we carried out various model runs with only the best results being shown here.

Moreover, to investigate problems related to over-fitting, which are commonly encountered when ANN-based models are developed, different numbers of hidden units were investigated. We evaluated the influence of the number of hidden units (neurons) in the final model performance, for training and testing horizons, based on the final value of the objective function (Eq. (2)). The results showed that for Case 2, the ANN operation model with four hidden neurons presented the best performance (highest value of objective function for the testing horizon), as shown in Fig. 10. We found this test important for improving the ANN operation model performance and robustness. For Case 1, the ENNIS's which presented the best performance were built with four hidden units, representing 21 and 25 parameters for the ENNIS using the averaged and observed inflow, respectively. The final number of neurons adopted for the ANN operation model of Case 2 is shown in Table 3. 
Table 3

Results after the operation by the M-5 curves and ENNIS models with different inflow information

\begin{tabular}{|c|c|c|c|c|}
\hline & \multicolumn{4}{|c|}{ Type of model } \\
\hline & M-5 & ENNIS & ENNIS & ENNIS \\
\hline Inflow information & - & Averaged & Observed & Predicted \\
\hline No. of units (I-H-O) & - & $7-4-5$ & $8-4-5$ & 8-2-5 \\
\hline No. of parameters & - & 57 & 61 & 33 \\
\hline \multicolumn{5}{|l|}{ Training } \\
\hline Average $E\left(W_{\text {ws }}\right)$ & 0.999 & 0.999 & 1.000 & 0.999 \\
\hline Average $E\left(W_{\text {oth }}\right)$ & 0.999 & 0.998 & 1.000 & 1.000 \\
\hline Average $E\left(Q_{w s}\right)$ & 0.998 & 0.998 & 1.000 & 1.000 \\
\hline Average $E\left(Q_{\text {oth }}\right)$ & 0.998 & 0.998 & 0.984 & 1.000 \\
\hline Average $E(\mathrm{hp})$ & 0.295 & 0.295 & 0.295 & 0.295 \\
\hline Average $E\left(Q_{\text {env }}\right)$ & 0.906 & 0.906 & 0.932 & 0.913 \\
\hline Aggregated object & 0.866 & 0.866 & 0.869 & 0.868 \\
\hline Number of failures & 13 & $\mathbf{0}$ & $\mathbf{0}$ & $\mathbf{0}$ \\
\hline Objective function & 0.855 & 0.866 & 0.869 & 0.868 \\
\hline Minimum & 0.168 & 0.671 & 0.712 & 0.733 \\
\hline Standard deviation & 0.083 & 0.059 & 0.056 & 0.056 \\
\hline \multicolumn{5}{|l|}{ Testing } \\
\hline Average $E\left(W_{\mathrm{ws}}\right)$ & 0.978 & 0.994 & 0.999 & 0.995 \\
\hline Average $E\left(W_{\text {oth }}\right)$ & 0.966 & 0.995 & 0.999 & 0.999 \\
\hline Average $E\left(Q_{w s}\right)$ & 0.978 & 0.992 & 0.999 & 0.999 \\
\hline Average $E\left(Q_{\text {oth }}\right)$ & 0.966 & 0.992 & 0.970 & 0.999 \\
\hline Average $E(\mathrm{hp})$ & 0.298 & 0.296 & 0.297 & 0.296 \\
\hline Average $E\left(Q_{\text {env }}\right)$ & 0.937 & 0.885 & 0.915 & 0.892 \\
\hline Aggregated object & 0.854 & 0.859 & 0.863 & 0.864 \\
\hline Number of failures & 24 & 4 & 10 & 14 \\
\hline Objective function & 0.820 & 0.854 & 0.849 & 0.844 \\
\hline Minimum & 0.185 & 0.574 & 0.684 & 0.634 \\
\hline Standard deviation & 0.112 & 0.070 & 0.065 & 0.064 \\
\hline
\end{tabular}

Note: (I-H-O) represents the number of units of the input, the hidden and the output layer, respectively. "Objective function" accounts for the final value of the objective function (higher values are better). "Minimum" means the minimum value found within an operation horizon for the aggregated value at each stage. "Standard deviation" means the standard deviation of all aggregated values within an operation horizon. "Number of failures" shows how many times (stages) the operation failed during a certain operation horizon.

\subsection{Results for Case 1: Single decision}

To demonstrate the optimality and efficiency of the proposed ENNIS, it was applied to a simple optimization/operation problem, with one decision variable, and its results were compared to those obtained by a DDP model. First, we optimized the reservoir with the deterministic DP (DDP), for which future inflows were considered to be exactly known and also considered two levels of discrete intervals, i.e., 5 and 50, for the decision variable. This low level of intervals (five) is frequently adopted in solving problems with DP that present more than one decision and one state variable. Fig. 9 shows a comparison of the results obtained for average energy pro-

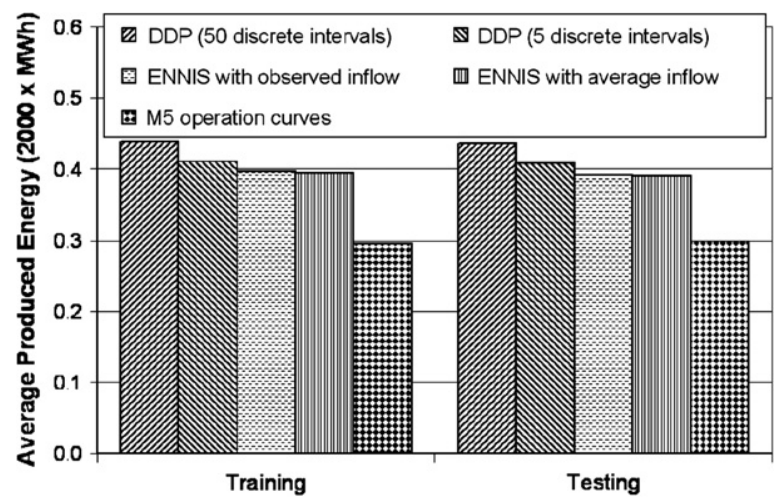

Fig. 9. Energy produced by different operational methods - Case 1 .

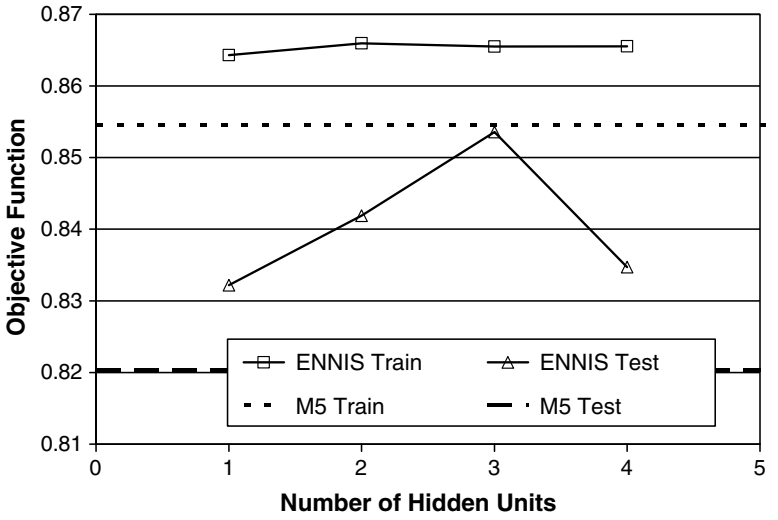

Fig. 10. Objective function value vs. number of hidden units.

duced by these two techniques, as well as the ones obtained by the M-5 rule curves. Accordingly, we can see that the results obtained by the proposed ENNIS model are able to generate an amount of average energy quite similar to the one obtained by the DDP models, especially for the situation with five discrete intervals. This clearly shows that the ENNIS can provide a good operational strategy for maximization of the operational objective (water release for energy production). In Fig. 9, only as an illustration, we also give the results obtained with the M-5 rule curves which are much lower than the ones obtained with the other two techniques.

For the results obtained for the testing horizon, also shown in Fig. 9, the reservoir was operated by the same developed ENNIS model with the same parameters obtained during the training phase, also considering averaged and observed inflow information as ANN input neuron. This shows the great robustness of the proposed intelligent system, as the results obtained were fairly similar to those by the DDP model. This is valid because the future inflows are not considered as input information of the ENNIS. On the contrary, the DDP considers known the future inflow sequence and it must be noted that we could not derive the operation strategies directly with deterministic DP models.

\subsection{Results for Case 2: Multiple decisions}

For Case 2, a more complex problem was formulated, which involved the operation of a system with five decision variables. For this case, the application of DDP models would be very complex or even infeasible, due to the curse of dimensionality. Therefore, results obtained by the ENNIS are compared just to the ones obtained by the current strategy, the M-5 rule curves. This case also intends to show the ability of the proposed intelligent system in handling various decision variables.

Figs. 11 and 12, together with Table 3, show the results obtained with the two operational strategies, M-5 and ENNIS, for the training and testing phase, respectively. These figures show the results obtained and three different formulations of ENNIS, which differ by the type of inflow information considered as input neuron. For the first case an ANN operation model is developed with seven input units. Then, for the next two formulations, an extra input unit is added to the network, corresponding, respectively, to predicted and observed inflow information, as shown in Fig. 7 as a dashedline circle.

For the training phase (Fig. 11) when considering the inflow information, either observed or predicted, we can see a slight improvement in the final value of the objective function (OF). Interestingly, for the testing phase (Fig. 12), the OF value for the ENNIS by only using 10-day historical averaged inflow, presented superior performance over the other formulations. This may be ex- 


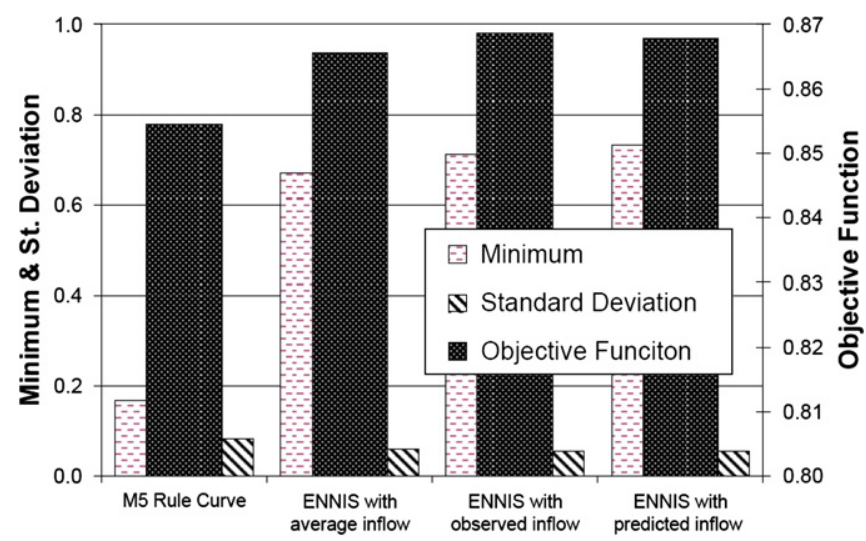

Fig. 11. Comparison of results by the M-5 and ENNIS operational strategies - training horizon.

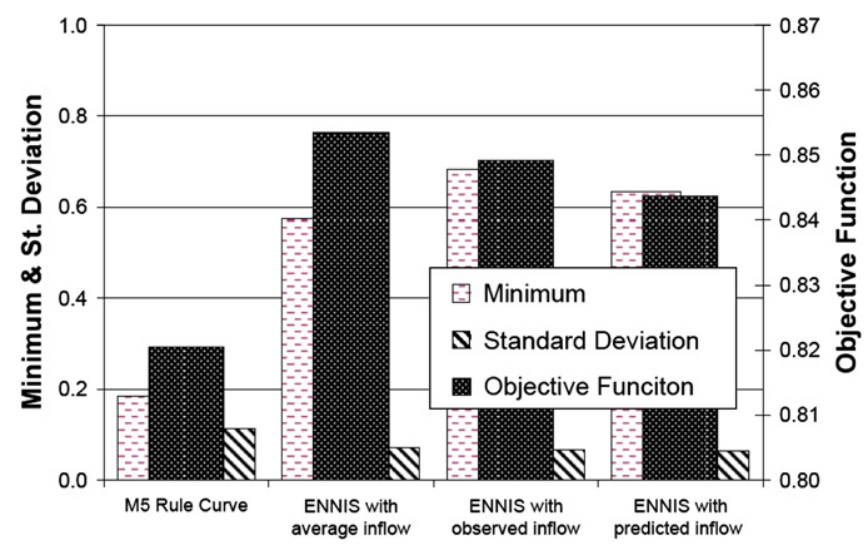

Fig. 12. Comparison of results by the M-5 and ENNIS operational strategies - testing horizon.

plained in several ways. First, as the reservoir presents low regulatory capability, due to its small total capacity, even knowing one-step ahead does not guarantee avoiding the failures of the operation during long dry periods. Another reason may be due to the enormous variation of inflow discharges, especially between low and dry periods, as a consequence of extreme precipitation events on the region caused by typhoons. In addition, probably the most important reason is related to the occurrence during the testing horizon of the most severe droughts within the historical data. Therefore, the ANN operation model was trained with no previous experience of such patterns.

For both operational horizons, the three ENNIS formulations resulted in superior performance than the M-5 curves, particularly when considering the number of failures, as can be seen in Table 3. The number of failures of the system represents the number of times the system failed to achieve certain conditions within a certain operational horizon, as already previously described. Table 3 also presents some of the statistical metrics of the aggregated values, such as the minimum and standard deviation, for training and testing horizons. These metrics, "minimum" and "standard deviation", may be seen as measures of robustness and volatility of the system operation, respectively. Also for these results, the three ENNIS showed to be superior to the M- 5 operation.

Also in Table 3, we can observe the averaged value of the evaluation function for each objective. This may give us a feeling of how the ENNIS is prioritizing the various objectives. This analysis gives us another insight into how to best adjust the evaluation
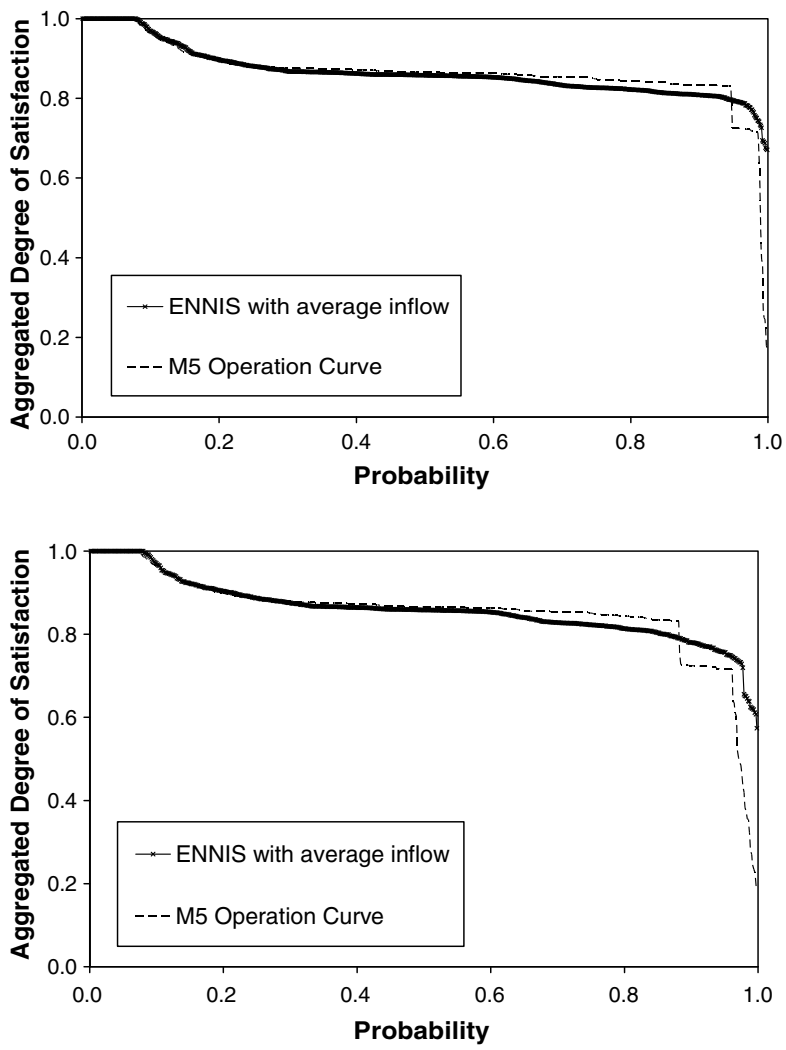

Fig. 13. Statistics of the aggregated value of the operational objectives: training and testing.

functions for achieving an efficient ANN operation model. We can also see in Fig. 13 a statistic of the results of the aggregation of the evaluated objectives obtained after the first ENNIS formulation. The shapes of the cumulative probability function for both ENNIS and $\mathrm{M}-5$ are quite similar for most of the percentage domain. Nevertheless, the ENNIS presented much better performance than the M-5 rule curves, avoiding failures (low values of the OF) related to the reservoir operation. These probabilistic curves were built considering the 864 and 612 aggregated values obtained for the training and testing horizons, respectively. This is another good proof of the robustness of the presented methodology and the built intelligent systems.

Figs. 14 and 15 show the final operating storage results obtained when considering the ENNIS ("Storage ENNIS") based on the ANN operation model with average inflow information, which required only 57 parameters (weights and bias) to be identified. Note that if we had used a pure GA to do the same job, we would need to optimize 4320 ( $=5$ decisions $\times 864$ time steps) and 3060 $(=5 \times 612)$ parameters, for the training and testing horizons, respectively, and even then we would not necessarily be able to derive any operational strategy for future use. For illustration, the results obtained by our intelligent operation system were compared to those from the present operational strategy ("Storage $M-5$ ") of Shihmen Reservoir. Note that in most cases when the M-5 operation resulted in failure, the ENNIS tended to depreciate the reservoir at a much slower pace, avoiding the risk involved in large depreciation of storage volumes.

Finally, we may see that the results after the developed intelligent operation system were very similar for both cases, training and testing horizons, which further demonstrates the consistence and robustness of the developed model as "optimal" operational strategies. This is not necessarily the case for the operation by 


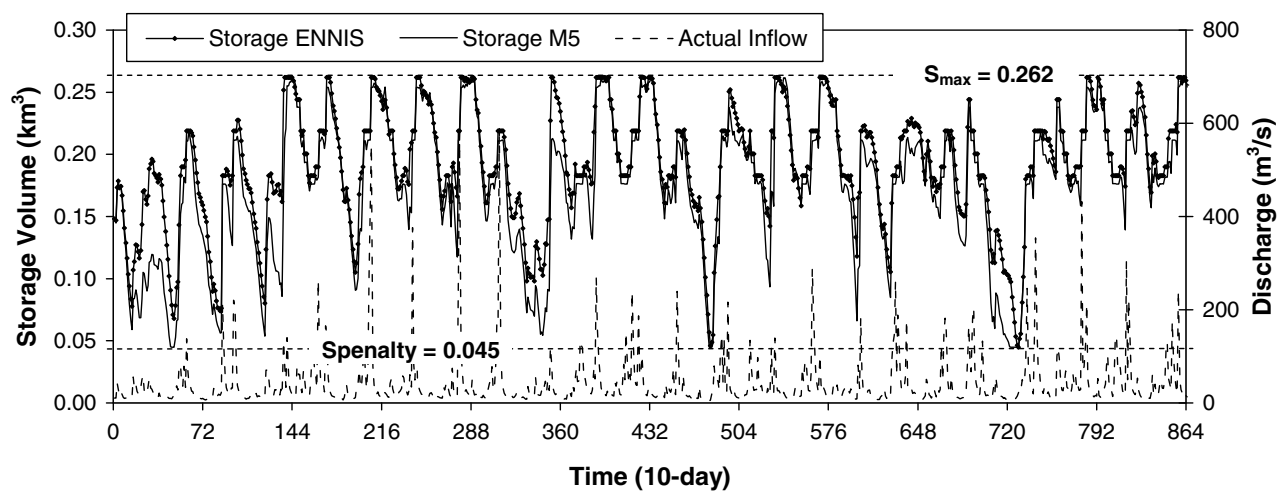

Fig. 14. Storage volumes operated by M-5 and ENNIS (training horizon).

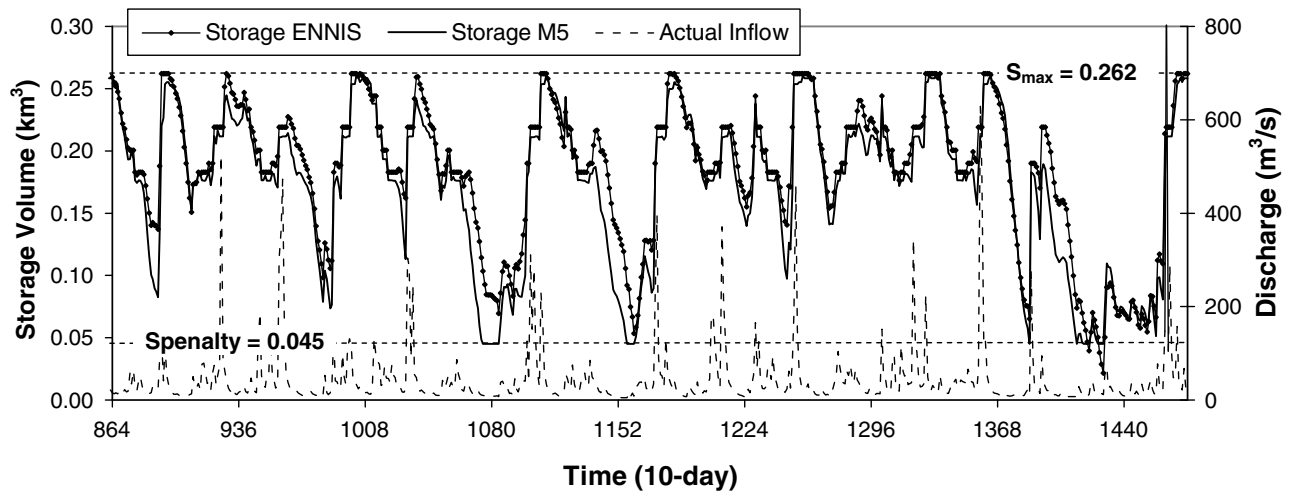

Fig. 15. Storage volumes operated by M-5 and ENNIS (testing horizon).

the M-5 strategy, as the results for the training and testing horizons periods were quite different from each other.

\section{Conclusions}

We developed a methodology for the construction of intelligent systems for reservoir operation, the Evolving Neural Network Intelligent System (ENNIS). The system is based on two popularly used artificial intelligence techniques: (i) ANN for constructing a multiple decision operation model and (ii) GA for training (optimizing) the ANN-based model. We have also fuzzified the multiple objectives of the reservoir as membership functions to be able to aggregate them together as a single objective.

First, the ENNIS was constructed and applied for a simple case, for which only one decision variable (released water) from Shihmen Reservoir, Taiwan, was derived. Its results were comparable to the results obtained by a deterministic dynamic programming (DPP), for which future inflows were considered to be exactly known. The results were fairly similar by both methods in both training and test phases. This clearly shows that the ENNIS can provide a good and robust operational strategy and can be a great potential for operational use. Then, another ENNIS was built and applied with success for a multi-variable decision making process, for the same case study reservoir with five variables. The GA model identified the weights of the ANN operation model, which after being applied to a testing dataset showed to be very robust, yielding better performances, in terms of the five objective defined in this study, than the ones by the present operational strategy, the M-5 rule curves.

Moreover, we have also investigated the influence on the performance of the operation model, by testing a different number of hidden units and different types of input and output variables. From this process, we identified the "best" set parameters for the final ANN operation model for the developed intelligent system. For the decision-taking process by the developed ENNIS, three types of information were used as input for the ANN model, beginning-of-period storage volume for the current stage, 10day average inflow information (historical, future observed and predicted values), and various water demands. Except for the actual future inflow, all these variables are readily available to operators at the time of operational decisions. So, the developed intelligent system has great potential to be extended for practical applications of reservoir operation. In addition, it is important to remember that GA models are capable of optimizing a great number of parameters, up to thousands. So that, even for large networks (taking a great number of decisions), we may be still able to apply the ENNIS methodology. On the other hand, one of the drawbacks of the proposed method may be related to the timing for training the ANN operation model, which is a characteristic of the GA optimization technique. Nevertheless, the proposed intelligent system may be trained off-line, and then applied for the real-time operation, without compromising its applicability.

Finally, due to the possibility to easily add extra input and output neurons to the ANN-based model, the proposed methodology shows great potential to incorporate other forecast models. This has been done here through the consideration of an extra input neuron representing a predicted future inflow into the reservoir. Moreover, the ENNIS showed to be able to take simultaneously a great number of decisions without compromising its optimality, as the obtained results showed to be consistent for both training and testing horizons. 


\section{Acknowledgement}

This paper is based on partial work supported by National Science Council, ROC (Grant No. NSC94-2313-B-002-088).

\section{References}

[1] ASCE Task Committee on Application of artificial neural networks in hydrology - artificial neural networks in hydrology. I: preliminary concepts. J Hydrol Eng 2000;5(2):115-23.

[2] ASCE Task Committee on Application of artificial neural networks in hydrology - artificial neural networks in hydrology. II: hydrological applications. J Hydrol Eng 2000;5(2):124-37.

[3] Cancelliere A, Giuliano G, Ancarani A, Rossi G. A neural network approach for deriving irrigation reservoir operating rules. Water Resour Mgmt 2002;16(1):71-88.

[4] Chandramouli V, Raman H. Multireservoir modeling with dynamic programming and neural network. J Water Resour Plan Mgmt 2001;127(2):89-98.

[5] Chang FJ, Chen L, Chang LC. Optimizing the reservoir operating rule curves by genetic algorithms. Hydrol Process 2005;19:2277-89.

[6] Chang YT, Chang LC, Chang FJ. Intelligent control for modeling of real-time reservoir operation, part II: artificial neural network with operating rule curves. Hydrol Process 2005;19(7):1431-44.

[7] Chang LC, Chang FJ. Intelligent control for modeling of real-time reservoir operation. Hydrol Process 2001;15(9):1621-34.

[8] Chang YM, Chang LC, Chang FJ. Comparison of static-feedforward and dynamic-feedback neural networks for rainfall-runoff modelling. J Hydrol 2004;290(3-4):297-311.

[9] Chaves P, Kojiri T. Deriving reservoir operational strategies considering water quantity and quality objectives by stochastic fuzzy neural networks. Adv Water Resour 2007;30(5):1329-41.

[10] Chaves P, Tsukatani T, Kojiri T. Operation of storage reservoir for water quality by using optimization and artificial intelligence techniques. Math Comput Simul 2004;67(4-5):419-32.

[11] Esogbue AO. Dynamic programming for optimal water resources systems analysis. New Jersey: Prentice Hall; 1989.
[12] Huang WC, Yuan LC, Lee CM. Linking genetic algorithm with stochastic dynamic programming to the long-term operation of multireservoir system. Water Resour Res 2002;38(12):1304, doi:10.1029/2001WR001122.

[13] Jain SK, Das A, Srivastava DK. Application of ANN for reservoir inflow prediction and operation. J Water Resour Plan Mgmt 1999;125(5):263-71.

[14] Johonson VM, Rogers L. Accuracy of neural network approximators in simulation-optimization. J Water Resour 2000;126(2):48-56.

[15] Kacprzyk J. Multistage fuzzy control, a model based approach to fuzzy control and decision making. Baltimore: John Wiley \& Sons; 1997.

[16] Karbowski A, Malinowski K, Niewiadomska-Szynkiewicz E. A hybrid analytic/ rule-based approach to reservoir system management during flood. Decision Supp Syst 2005;38:599-610.

[17] Kurosh M. Artificial neural networks and intelligent information processing. In: Proceedings of the 1st international workshop on artificial neural networks and intelligent information processing, ANNIIP 2005, Barcelona, Spain; 2005.

[18] Labadie JW. Optimal operation of multireservoir systems: state-of-the-art review. J Water Resour Plan Mgmt 2004;130(2):93-111.

[19] Lin CL, Su HW. Intelligent control theory in guidance and control system design: an overview. Proc Nat Sci Council ROC 2000;24(1):15-30.

[20] Maier HR, Dandy GC. Neural network based modeling of environmental variables: a systematic approach. Math Comput Model 2001;33:669-82.

[21] Olsson J, Uvo CB, Jinno K, Kawamura A, Nishiyama K, Koreeda N, et al. Neural networks for rainfalling forecasting by atmospheric downscaling. J Hydrol Eng 2004;9(1):1-11.

[22] Peng RG, Wu RS, Bi LJ, Wen PW. Discussing the protected and nurtured flows in Taiwan. Water Resources Management Seminar, Taiwan; 2005.

[23] Ponnambalam K, Karray F, Mousavi SJ. Minimizing variance of reservoir system operations benefits using soft computing tools. Fuzzy Sets Syst 2003;139:451-61.

[24] Raman H, Chandramouli V. Deriving a general operating policy for reservoir using neural network. J Water Resour Plan Mgmt 1996;122(5):342-7.

[25] Russell SJ, Norvig P. Artificial intelligence: a modern approach. 2nd ed. Upper Saddle River (NJ): Prentice Hall; 2003 [ISBN: 0-13-790395-2].

[26] Sharif M, Wardlaw R. Multireservoir systems optimization using genetic algorithms: case study. J Comput Civil Eng 2000;14(4):255-63.

[27] Westphal KS, Vogel RM, Kirshen P, Chapra SC. Decision support system for adaptive water supply management. J Water Resour Plan Mgmt 2003;129(3):165-77.

[28] Yao X. Evolving artificial neural networks. Proc IEEE 1999;87(9):1423-47. 